\title{
Immunopotentiating Activity of Dendrobium Species in Mouse Splenocytes
}

\author{
David T. W. Lau, Michel K. T. Poon, Hoi Yan Leung, Kam Ming Ko \\ Division of Life Science, Hong Kong University of Science \& Technology, Hong Kong, China \\ E-mail: bcrko@ust.hk \\ Received April 4, 2011; revised April 25, 2011; accepted May 13, 2011
}

\begin{abstract}
This study aimed to explore a pharmacological activity marker for quality assurance of Dendrobium species. The immunopotentiating activity in aqueous extracts prepared from four Dendrobium species, including $D$. officinalis, was assessed by an in vitro assay of concanavalin A (Con A)-stimulated proliferation of mouse splenocytes. Four samples of commercially available Dendrobii Caulis were also analyzed for comparison. The results indicated that the aqueous extract of $D$. officinalis produced immunopotentiating action, as evidenced by the increase in Con A-stimulated proliferation of mouse splenocytes, with the extent of stimulation being more prominent than those of other tested Dendrobium species and Dendrobii Caulis samples. In conclusion, an in vitro immunopotentiation assay may be used for assessing the pharmacological activity of Dendrobium species. The finding that $D$. officinalis produced a more potent immunopotentiating action is consistent with its "yin-nourishing" action in Chinese medicine, which is more effective than other Dendrobium species in clinical use.
\end{abstract}

Keywords: Dendrobium offincalis, Dendrobii Caulis, Splenocyte Proliferation, Concanavalin A

\section{Introduction}

Dendrobium species was regarded as a first-rate herb in Shen-nong Bencao Jing (Canon on Medicinal Herbs, 200 B.C.) and has a long history of usage in China. Over the past few decades, the dried stem of Dendrobium species (Dendrobii Caulis, also called Shi Hu in Chinese) has been used for clearing "heat" and nourishing "yin" in the practice of Chinese medicine [1]. A recent study showed that the administration of an aqueous extract of Dendrobium candidum ameliorated the dry-mouth symptom in patients suffering from Sjögren syndrome [2]. Pharmacological studies have demonstrated that the aqueous extract and/or polysaccharides of Dendrobii Caulis possess antioxidant [3,4], immuno-stimulating [5-7], antitumor [8,9], anti-microbial [9], anti-hyperlipidemia [10] and anti-hyperglycemic [11] activities. The widespread application of Dendrobii Caulis has raised a concern regarding the identity of Dendrobium species as herbal source. In this regard, the record of Dendrobii Caulis in Chinese Pharmacopoeia has been constantly amended over the past 20 years. While five Dendrobium species, including $D$. loddigesii Rolfe, $D$. fimbriatum Hook and $D$. nobile Lindl., were listed in the 1995 and 2000 editions, a more extended list of Dendrobium species was generated in the 2005 edition. As such, up to 74 Dendrobium species can be used as Dendrobii Caulis in China. In the 2010 edition of Chinese Pharmacopoeia [12], D. Officinalis has been singled out as a distinct herb originated from $D$. officinale Kimura et Migo. Not surprisingly, $D$. Officinalis is relatively scarce in abundance and deemed to possess a stronger therapeutic potential than other Dendrobium species [13,14].

As more than 45 Dendrobium species have been identified to possess therapeutic potential [15-18], the development of chemical and DNA fingerprinting methods for species authentication, particularly for those recorded in Chinese Pharmacopoeia, has been an area of intensive research [19-26]. In addition to chemical markers, the measurement of pharmacological activity may offer a complementary and functionally relevant quality assessment for herbal materials [27]. In this connection, "yinnourishing” tonic herbs (including Herba Dendrobii) have been found to produce immunopotentiating effect on mouse splenocytes both in vivo and in vitro [28]. In the present study, using an in vitro assay of concanavalin A (Con A)-stimulated proliferation of mouse splenocytes, we assessed the immunopotentiating activity of aqueous 
extracts prepared from four Dendrobium species including $D$. Officinalis, with an objective of exploring a pharmacological activity marker for quality assurance. Four samples of commercially available Dendrobii Caulis were also analyzed for comparison.

\section{Materials and Methods}

\subsection{Chemicals and Plant Materials}

RPMI-1640 medium, heat inactivated fetal bovine serum (FBS) and Con A were purchased from Sigma Chemical Co. (St. Louis, MO, USA). MTT (3-[4,5-dimethylthiazol2-yl]-2,5-diphenyl tetrazolium bromide)-based cell proliferation kit was purchased from Boehringer Mannheim Gmbh (Mannheim, Germany). All other chemicals were of analytical grade. Four Dendrolium species, namely, D. loddigesii Rolfe, D. fimbriatum Hook, D. nobile Lindl. and $D$. officinalis Kimura et Migo, were obtained from an ornamental flora shop in Hong Kong, and they were authenticated by floral structure with reference to China Flora. Four samples of Dendrobium Caulis were randomly bought from local herbal stores. Voucher specimens of Dendrobium plants and Dendrobii Caulis have been deposited in the Division of Life Science, The Hong Kong University of Science \& Technology (HKUST), Hong Kong SAR, China.

\subsection{Plant/Herbal Extraction}

Fresh stems of Dendrobium plants or Dendrobii Caulis (i.e., dried stems of Dendrobium species) were cut into small pieces and extracted by 10 volumes (w/v) of double distilled water at $60^{\circ} \mathrm{C}$ for $2 \mathrm{~h}$. The extraction procedure was repeated once, and the pooled aqueous extracts were dried by lyophilization. The lyophilized powders (i.e. Dendrobium extracts) were stored at $-20^{\circ} \mathrm{C}$ prior to use for experiment.

\subsection{Measurement of in Vitro Immunopotentiating Activity in Con A-Stimulated Mouse Splenocytes}

Procedures involved in isolation of mouse splenocytes were conducted under aseptic conditions. Splenic tissue obtained from adult female ICR mice (25 - 28 g) was teased with a plastic syringe in a culture dish $(60 \mathrm{~mm})$ containing $10 \mathrm{~mL}$ of RPMI-1640 medium, and it was gently strained through a 200 mesh stainless steel sieve to remove clumps to produce a cell suspension. The product was then left to stand on ice for 5 min to remove tissue fragments. The cell suspension was centrifuged at $600 \times \mathrm{g}$ for $10 \mathrm{~min}$ and washed 3 times with RMPI-1640 medium, and it was finally resuspended in RPMI-1640 medium supplemented with $10 \%$ FBS at a concentration of $1 \times 10^{7}$ viable cells $/ \mathrm{mL}$. The viability of isolated splenocytes, as determined by Trypan blue exclusion test, was found to be higher than 95\%. Mouse splenocytes were cultured in medium with Con A in the presence or absence of Dendrobium extract in 96-well microtiter plates (flat bottom) in a final volume of $100 \mu \mathrm{L}$. Con A (prepared in phosphate buffered saline) was added at final concentrations of $0.5,1$ and $2 \mu \mathrm{g} / \mathrm{mL}$, respectively. Aliquots of respective Dendrobium extract $(10 \mu \mathrm{L}$ in aqueous solution) were added at increasing final concentrations ranging from 1.6 to $100 \mu \mathrm{g} / \mathrm{mL}$. Control wells were added with $10 \mu \mathrm{L}$ of sterilized double-distilled water only. Splenocytes were then cultured for $72 \mathrm{~h}$ at $37^{\circ} \mathrm{C}$ in a humidified atmosphere of $5 \% \mathrm{CO}_{2}$ in air. At the end of the culture period, the extent of splenocyte proliferation was determined colorimetrically by MTT-based cell proliferation assay. An aliquot $(10 \mu \mathrm{L})$ of MTT labeling reagent was added to each well under dark conditions. After $4 \mathrm{~h}$ of incubation, $100 \mu \mathrm{L}$ of solubilization buffer (10\% SDS in $0.01 \mathrm{M} \mathrm{HCl}$ ) was added, and the mixtures were incubated in $5 \% \mathrm{CO}_{2}$ at $37^{\circ} \mathrm{C}$ overnight for dissolving the colored crystals. The extent of splenocyte proliferation was determined by measuring the absorbance at $570 \mathrm{~nm}$ using Victor $\mathrm{V}^{2}$ Multi-label Counter (Perkin Elmer, Turku, Finland). Stimulation index (SI) was calculated using the equation: $\mathrm{SI}=$ mean absorbance of cells stimulated with Con A/ mean absorbance of cells not stimulated with Con A. The extent of Con A-stimulated proliferation of isolated splenocytes was estimated by computing the area under the curve $\left(\mathrm{AUC}_{1}\right)$ of the graph plotting stimulatory indices against Con A concentrations. Data of $\mathrm{AUC}_{1}$ were expressed as the percentage of non-Dendrobium extract-treated control, and $\mathrm{AUC}_{2}$ was computed from a graph plotting percentages of control against tested concentrations of Dendrobium extract. The immunopotentiating activity was expressed as the difference in $\mathrm{AUC}_{2}$ values between the Dendrobium extract-untreated control and Dendrobium-treated group.

\subsection{Measurements of Interleukin-2 (IL-2), IL-6 and Interferon- $\gamma($ INF- $\gamma)$ Levels}

Isolated mouse splenocytes were cultured with Dendrobium extract $(25,50,100 \mu \mathrm{g} / \mathrm{mL}$, final concentration) in the presence or absence of Con A ( $2 \mu \mathrm{g} / \mathrm{mL})$ for $24 \mathrm{~h}$. IL-2, IL-6 and INF- $\gamma$ levels in the culture medium was then measured using ELISA kits (Invitrogen ${ }^{\mathrm{TM}}$ Mouse IL-2 ELISA kit, Invitrogen, Frederick, MD, USA; Invitrogen $^{\mathrm{TM}}$ Mouse IL-6 ELISA kit, Invitrogen, Camarillo, CA, USA; ELISA ${ }^{\mathrm{PRO}}$ kit for mouse IFN- $\gamma, \mathrm{MABTECH}$, 
Inc., Cincinnati, OH, USA).

\section{Results and Discussion}

As shown in Figure 1, Con A-stimulated the proliferation of mouse splenocytes, as indicated by a dose-dependent increase in stimulatory index. While the aqueous extract of $D$. officinalis (DO) alone did not produce any detectable effect on mouse splenocyte proliferation (data not shown), it caused a dose-dependent enhancement in Con A-stimulated proliferation of mouse splenocytes in vitro. When the extent of Con A-stimulated proliferation was estimated by computing the AUC of the Con A dose-response curve (AUC $)_{1}$ ) and then by the AUC of the DO dose-response curve $\left(\mathrm{AUC}_{2}\right)$, the extent of Con A-stimulated proliferation of splenocytes induced by DO was found to be increased by $23 \%$, when compared with the DO-untreated and Con A-stimulated control. Among the tested aqueous extracts of Dendrobium species, $D$. nobile (DN) and D. loddigesii (DL) produced a slight enhancing effect on Con A-stimulated proliferation (1.2 and $3.6 \%$, respectively), whereas D. fimbriatum (DF) caused a mild suppressive effect (-6.4\%) (Figure 2). Among the four tested Dendrobii Caulis samples $\left(\mathrm{DC}_{1-4}\right)$, $\mathrm{D}_{3}$ and $\mathrm{D}_{4}$ showed a slight enhancing effect on Con A-



Figure 1. Effect of $D$. officinalis extract on Con A-stimulated proliferation of mouse splenocytes. Isolated mouse splenocytes were incubated with increasing concentrations of Con $A(0-2 \mu \mathrm{g} / \mathrm{mL})$ in the absence or presence of an aqueous extract of $D$. officinalis (DO) $(1.6-100 \mu \mathrm{g} / \mathrm{mL})$, as described in Materials and methods. Data of two representative concentrations $(6.25$ and $100 \mu \mathrm{g} / \mathrm{mL})$ of DO are shown. Values given are mean \pm S.D., with data obtained from triplicate assay samples. *Significantly different from Dendrobium extract-untreated control.

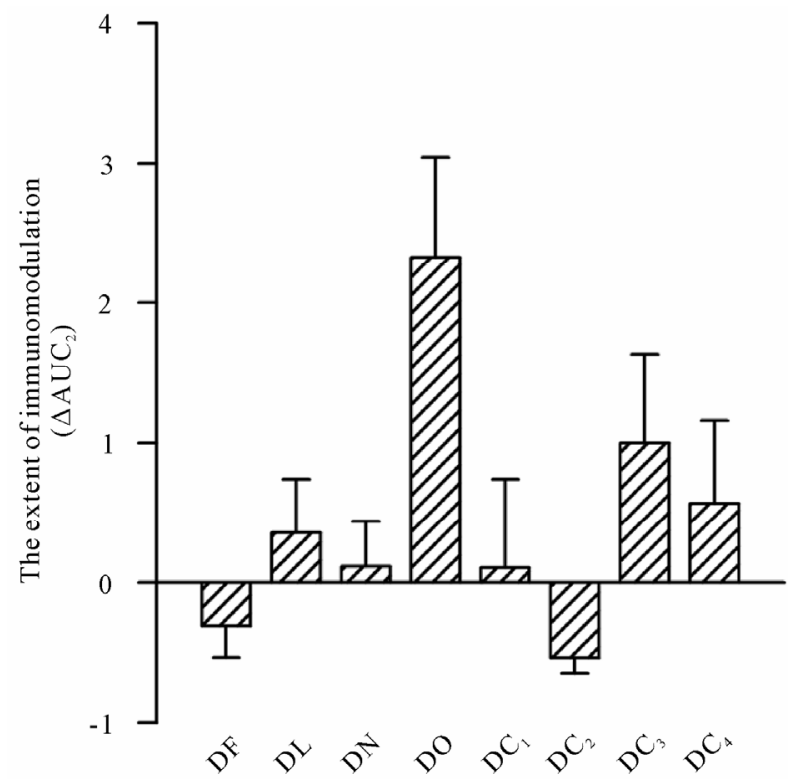

Figure 2. Immunopotentiating activity of Dendrobium extracts in Con A-stimulated mouse splenocytes. Isolated mouse splenocytes were incubated with Con $A$ and Dendrobium extracts $(D$. fimbriatum, DF; $D$. loddigesii, DL; $D$. nobile, DN; DO; Dendrobium Caulis, $\left.\mathrm{DC}_{1-4}\right)$, as described in Figure 1. The extent of immunopotentiation was estimated and data were expressed as $\Delta \mathrm{AUC}_{2}$, according to the computation procedure described in Materials and methods. The value of $\mathrm{AUC}_{2}$ for Dendrobium extract-untreated and Con A-stimulated control was 10. Values given are mean \pm S.D., with data obtained from triplicate assay samples.

stimulated proliferation of mouse splenocytes (5.4\% and $10 \%$ ), but $\mathrm{D}_{1}$ showed undetectable and $\mathrm{D}_{2}$ produced suppressive effect $(-5.4 \%)$.

Splenocyte proliferation is a complex event that involves interaction of cytokines such as IL-1 and IL-2 and expression of their receptors [29]. The ability of Dendrobium extract to enhance Con A-stimulated splenocyte proliferation may therefore be mediated by the modulation of cytokine expression and/or function. As shown in Figure 3(a), Con A caused an increase in IL-2 production in cultured mouse splenocytes (Figure 3(b)). DO enhanced the Con A-stimulated IL-2 production in mouse splenocytes, with the extent of stimulation being $18 \%$ at $100 \mu \mathrm{g} / \mathrm{mL}$. In addition, Con A also stimulated IL-6 and INF- $\gamma$ production in mouse splenocytes (Figure 3(b), (c)), and the Con A-stimulated IL-6 and INF- $\gamma$ production were enhanced by DO, with the degree of stimulation being $55 \%$ and $24 \%$, respectively, at a concentration of $100 \mu \mathrm{g} / \mathrm{mL}$. IL-6 and INF- $\gamma$, which are cytokines secreted by $\mathrm{T}$ helper cells, can activate neutrophils [30-32] and macrophages [33-35], respectively. The enhancement of Con A-stimulated splenocyte proliferation by DO was associated with the stimulation of cytokine secretion, thereby producing a generalized immunopoten- 


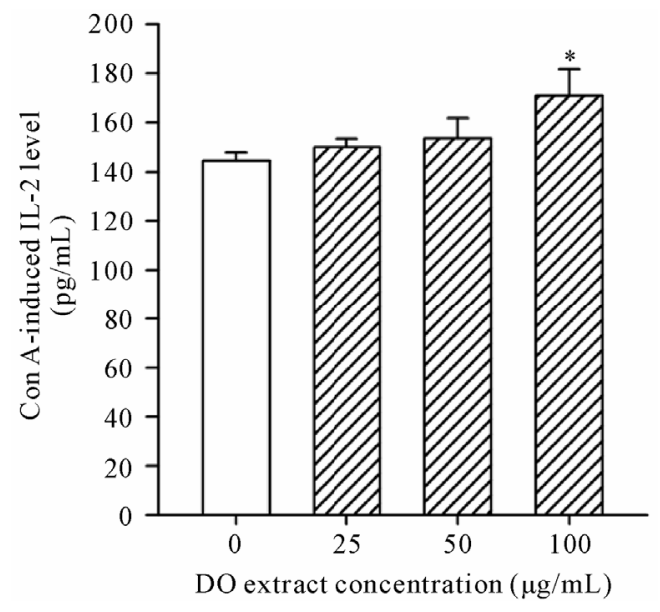

(a)

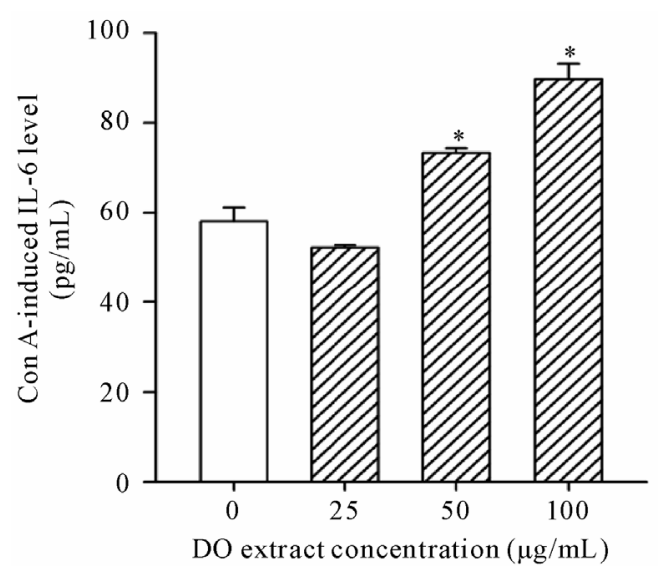

(b)

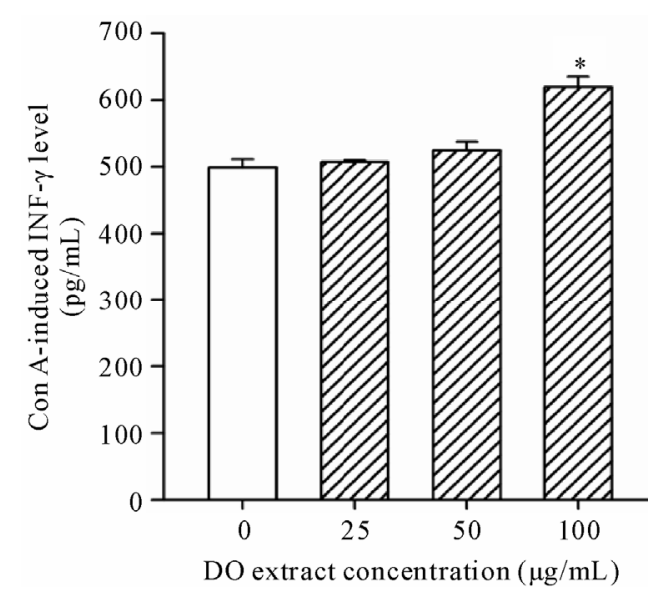

(c)

Figure 3. Effects of $D$. officincalis extract on cytokine levels in Con A-stimulated mouse splenocytes. Mouse splenocytes were treated with Con $A(2 \mu \mathrm{g} / \mathrm{mL})$ and DO $(25-100 \mu \mathrm{g} / \mathrm{mL})$. (a) IL-2, (b) IL-6 and (c) INF- $\gamma$ evels were measured in the cultured medium. Values given are mean \pm S.D., with data obtained from triplicate assay samples. *Significantly different from the DO-untreated and Con A-stimulated control. tiating effect.

An earlier study in our laboratory has shown that "yin-nourishing" Chinese tonic herbs can enhance the Con A-stimulated mitogenic response of mouse splenocytes both in vitro and ex vivo [28]. In the present study, the finding that $D$. officinalis produced a marked immunopotentiating action further supports the use of splenocyte mitogenic assay for the assessment of "yin-nourishing” action. Interestingly, among the tested Dendrobium species and Dendrobii Caulis samples, D. officinalis is the most potent in immunopotentiation. The finding also provides a pharmacological basis for the adoption of $D$. officinalis as a premium Dendrobii Caulis for "yin-nourishing" in the latest edition of Chinese Pharmacopoeia [12]. The observation that DO obtained from water extraction at room temperature rather than $60^{\circ} \mathrm{C}$ did not produce stimulatory effect on mouse splenocyte proliferation suggests the involvement of polysaccharides in immunopotentiating action (unpublished data).

\section{Conclusions}

The results indicated that the aqueous extract of $D$. officinalis produced immunopotentiating action in mouse splenocytes, which was more prominent than those of other tested Dendrobium species and Dendrobii Caulis samples. The in vitro immunopotentiation assay may be used for assessing the pharmacological activity of Dendrobium species. The finding that $D$. officinalis produced a more potent immunopotentiating action is consistent with its "yin-nourishing" action in Chinese medicine, which is more effective than other Dendrobium species in clinical use.

\section{References}

[1] Chinese Pharmacopoeia Commission, "Pharmacopoeia of the People's Republic of China,” China Medical Science Press, Beijing, 2005.

[2] L. Xiao, T. B. Ng, Y. B. Feng, T. Yao, J. H. Wong, R. M. Yao, L. Li, F. Z. Mo, Y. Xiao, P. C. Shaw, Z. M. Li, S. C. W. Sze and K. Y. Zhang, "Dendrobium candidum Extract Increases the Expression of Aquaporin-5 in Labial Glands from Patients with Sjögren's Syndrome,” Phytomedicine, Vol. 18, No. 2-3, 2011, pp. 194-198.

[3] A. Luo, X. He, S. Zhou, Y. Fan, T. He and Z. Chun, “In Vitro Antioxidant Activities of a Water-Soluble Polysaccharide Derived from Dendrobium nobile Lindl. Extracts," International Journal of Biological Macromolecules, Vol. 45, No. 4, 2009, pp. 359-363. doi:10.1016/j.ijbiomac.2009.07.008

[4] X. Zhang, J. K. Xu, N. L. Wang, K. Hiroshi, X. S. Yao and Z. Wang, "Studies on Antioxidant Activity of Bibenzyls and Phenolic Components from Dendrobium no- 
bile," Chinese Pharmaceutical Journal, Vol. 43, 2008, pp. 829-832.

[5] J. H. Wang, J. P. Luo, X. Q. Zha and B. J. Feng, “Comparison of Antitumor Activities of Different Polysaccharide Fractions from the Stems of Dendrobium nobile Lindl," Carbohydrate Polymers, Vol. 79, No. 1, 2010, pp. 114-118. doi:10.1016/j.carbpol.2009.07.032

[6] X. Q. Zha, J. P. Luo, S. Z. Luo and S. T. Jiang, "Structure Identification of a New Immunostimulating Polysaccharide from the Stems of Dendrobium huoshanense," Carbohydrate Polymers, Vol. 69, No. 1, 2007, pp. 86-93.

[7] X. Q. Zha, J. P. Luo and S. T. Jiang, "Induction of Immunomodulating Cytokines by Polysaccharides from Dendrobium huoshanense," Pharmaceutical Biology, Vol. 45, No. 1, 2007, pp. 71-76. doi:10.1080/13880200601028420

[8] J. H. Wang, X. Q. Zha, J. P. Luo and X. F. Yang, “An Acetylated Galactomannoglucan from the Stems of Dendrobium nobile Lindl," Carbohydrate Research, Vol. 345, No. 8, 2010, pp. 1023-1027. doi:10.1016/j.carres.2010.03.005

[9] D. Uma, S. Selvi, D. Devipriya, S. Murugan and S. Suja, "Antitumor and Antimicrobial Activities and Inhibition of in-Vitro Lipid Peroxidation by Dendrobium nobile," African Journal of Biotechnology, Vol. 8, No. 10, 2009, pp. 2289-2293.

[10] X. Y. Li, Q. H. Gong, Q. Wu, Y. F. Lu, F. Jin, Y. F. Li and J. S. Shi, "Effects of Dendrobium nobile Polyose on Hyperlipemia and Liver Fatty Degeneration in Rats," Chinese Pharmaceutical Journal, Vol. 45, 2010, pp. 1142-1144.

[11] Y. Zhao, Y. O. Son, S. S. Kim, Y. S. Jang and J. C. Lee, "Antioxidant and Anti-Hyperglycemic Activity of Polysaccharide Isolated from Dendrobium chrysotoxum Lindl," Journal of Biochemistry and Molecular Biology, Vol. 40, No. 5, 2007, pp. 670-677. doi:10.5483/BMBRep.2007.40.5.670

[12] Chinese Pharmacopoeia Commission, "Pharmacopoeia of the People’s Republic of China,” China Medical Science Press, Beijing, 2010.

[13] X. Lin, C. W. Sze, Y. Tong, Z. Zhang, Y. Feng, J. P. Chen, T. B. Ng, L. Xiao, P. Shaw and K. Y. Zhang, "Protective Effect of Dendrobium officinale Polysaccharides on Experimental Sjögren's Syndrome,” Journal of Complementary and Integrative Medicine, Vol. 7, No. 1, 2010, Article 14. doi:10.2202/1553-3840.1342

[14] Y. Wu and J. Si, "Present Status and Sustainable Development of Dendrobium officinal Industry," China Journal of Chinese Materia Medica, Vol. 35, No. 15, 2010, pp. 2033-2037.

[15] W. L. Sha and J. Y. Luo, "Study of the Chinese drug Shi$\mathrm{Hu}$ (Dendrobium). I. Investigation of Botanical Origin and the Drug," Acta Pharmaceutica Sinica, Vol. 15, No. 6, 1980, pp. 351-357.

[16] G. X. Ma, G. J. Xu, L. S. Xu and M. F. Li, "Survey and Identification of Commercial Samples of Shihu (Dendrobium Sw.) (III)," Chinese Traditional and Herbal Drugs, Vol. 20, 1995, pp. 370-373.
[17] J. Liu, T. He and Z. Chun, "DNA Molecular Identification of Herba Dendrobii and Its Adulterant Species Based on ITS Sequence Analysis," China Journal of Chinese Materia Medica, Vol. 34, No. 22, 2009, pp. 2853-2856.

[18] Z. Q. Yuan, J. Y. Zhang and T. Liu, "Phylogenetic Relationship of China Dendrobium Species Based on the Sequence of the Internal Transcribed Spacer of Ribosomal DNA,” Biologia Plantarum, Vol. 53, No. 1, 2009, pp. 155-158. doi:10.1007/s10535-009-0024-0

[19] J. Liu, T. He and Z. Chun, "Advance in Application of DNA Molecular Markers on Dendrobium," Chinese Journal of Applied and Environmental Biology, Vol. 14, No. 6, 2008, pp. 855-862.

[20] Y. P. Li, C. Qing, T. T. Fang, Y. Liu and Y. G. Chen, "Chemical Constituents of Dendrobium chrysotoxum," Chemistry of Natural Compounds, Vol. 45, No. 3, 2009, pp. 414-416. doi:10.1007/s10600-009-9329-7

[21] H. J. Ou, J. L. Cheng, X. X. Li, R. T. Zhan, Y. Z. Liang, H. Xu and P. Yan, "HPLC Fingerprint of Flavonoids and Phenols of Dendrobium nobile," Journal of Chinese Medicinal Materials, Vol. 32, No. 6, 2009, pp. 871-874.

[22] S. G. Shao, L. Han, Y. H. Ma, J. Shen, W. C. Zhang and X. Y. Ding, "Analysis and Authentication of cpDNA psbAtrnH Regions of Dendrobium Species of Fengdous,” Acta Pharmaceutica Sinica, Vol. 44, 2009, pp. 1173-1178.

[23] S. J. Wu, Y. S. Liu, T. W. Chen, C. C. Ng, W. S. Tzeng and Y. T. Shyu, "Differentiation of Medicinal Dendrobium Species (Orchidaceae) Using Molecular Markers and Scanning Electron Microscopy," Journal of Food and Drug Analysis, Vol. 17, No. 6, 2009, pp. 474-488.

[24] H. H. Fan, T. C. Li, J. Qiu, Y. Lin and Y. P. Cai, "Comparison of SRAP and RAPD Markers for Genetic Analysis of Plants in Dendrobium sw.," Chinese Traditional and Herbal Drugs, Vol. 41, No. 4, 2010, pp. 627-632.

[25] S. G. Feng, X. Hu, H. Y. Zhao and H. Z. Wang, "Application of DNA Molecular Marker in Study on Dendrobium officinale," Chinese Traditional and Herbal Drugs, Vol. 41, 2010, pp.499-502.

[26] D. Xue, S. Feng, H. Zhao, H. Jiang, B. Shen, N. Shi, J. Lu, J. Liu and H. Wang, " The Linkage Maps of Dendrobium Species Based on RAPD and SRAP Markers," Journal of Genetics and Genomics, Vol. 37, No. 3, 2010, pp. 197-204. doi:10.1016/S1673-8527(09)60038-2

[27] K. M. Ko and K. M. Ng, "Processing Control of Chinese Herbal Products: An Ultimate Approach for Quality Assurance,” Asia-Pacific Biotechnology News, Vol. 8, No. 23, 2004, pp. 1338-1341.

[28] T. K. Yim and K. M. Ko, “Antioxidant and ImmunoModulatory Activities of Chinese Tonifying Herbs, in Vitro and ex Vivo," Pharmaceutical Biology, Vol. 40, No. 5, 2002, pp. 329-335. doi:10.1076/phbi.40.5.329.8457

[29] K. A. Smith, "Interleukin-2: Inception, Impact, and Implications,” Science, Vol. 240, No. 4856, 1988, pp. 11691176. doi:10.1126/science.3131876

[30] J. Parkin and B. Cohen, "An Overview of the Immune System,” The Lancet, Vol. 357, No. 4856, 2001, pp. 17771789. 
[31] S. D. Kobayashi, J. M. Voyich, C. Burlak and F. R. DeLeo, "Neutrophils in the Innate Immune Response," Archivum Immunologiae et Therapia Experimentalis (Warsz), Vol. 53, No. 6, 2005, pp. 505-517.

[32] C. Guzmán, C. Hallal-Calleros, L. López-Griego and J. Morales-Montor, "Interleulin-6: A Cytokine with a Pleiotropic Role in the Neuroimmunoendocrine Network," The Open Neuroendocrinology Journal, Vol. 3, 2010, pp. 152-160.

[33] S. Gordon, "Alternative Activation of Macrophages," Nature Review Immunology, Vol. 3, 2003, pp. 23-35.

\section{doi:10.1038/nri978}

[34] D. M. Mosser, "The Many Faces of Macrophage Activation,” Journal of Leukocyte Biology, Vol. 73, No. 2, 2003, pp. 209-212. doi:10.1189/jlb.0602325

[35] C. Pujol, J. P. Grabenstein, R. D. Perry and J. B. Bliska, "Replication of Yersinia pestis in Interferon Gamma-Activated Macrophages Requires ripA, a Gene Encoded in the Pigmentation Locus," Proceedings of the National Academy of Sciences, Vol. 102, No. 36, 2005, pp. 1290912914. 